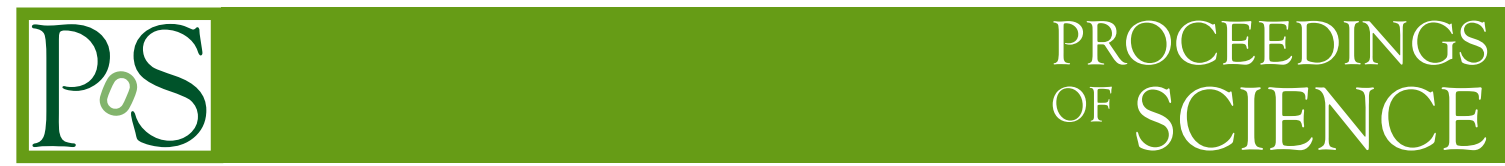

\title{
Coherent lattice QCD
}

\author{
Ivan Horváth* \\ Department of Physics and Astronomy, University of Kentucky, Lexington, KY 40503 \\ E-mail: horvathepa.uky.edu
}

We discuss a proposal for the construction of lattice QCD with gauge action, fermionic action, $\theta$-term, and the operators all based on the lattice Dirac operator $D$ with exact chiral symmetry. The simplest regularization of this type uses the proposition that the classical limit of scalar gauge density associated with trace of $D$ is (up to an additive constant) proportional to $\operatorname{tr} F^{2}$, while the corresponding operator is local. More general formulations from this class are considered with the aim of exposing interrelations between gauge and fermionic aspects of QCD which are otherwise hidden in generic formulations. Possible utility of these formulations for exploring QCD vacuum structure is emphasized.

XXIVth International Symposium on Lattice Field Theory

July 23-28, 2006

Tucson, Arizona, USA

${ }^{*}$ Speaker. 


\section{Vacuum Structure Motivation}

When studying QCD vacuum structure in the path integral formalism, the basic issue we face is the apparent clash of two tendencies. On one side there is randomness that is inherent in the definition of the theory, while on the other side there is space-time order that we seek in order to understand the vacuum. The root of the problem is largely related to the fact that we cannot completely eliminate one or the other. Indeed, eliminating the randomness seems to require abandoning the field theory description of strong interactions - a step that is difficult to contemplate otherwise. On the other hand, the absence of the need for the element of space-time order would imply that vacuum cannot be understood in the language of path integrals. This means that the analysis of some equilibrium configuration $U$ in lattice-regularized theory should not only be able to distinguish randomness from order, but it should also separate the randomness that is physically necessary from one that is useless, and can thus be eliminated. This is a highly non-trivial problem.

The proper formulation of the tasks qualitatively described above requires the use of concepts well established in the information theory and the theory of computation. A particular fusion of these subjects known as algorithmic information theory seems to be particularly relevant [1]. At the same time, once the underlying issues are properly identified, one can also attempt a "physicist's approach" to the problem, trying to take advantage of possible shortcuts offered by the proper physical insight. Following this path within the "Bottom- $U p$ " approach to QCD vacuum structure $[2,1]$, we will rely on two guiding points. (1) Since truly random fluctuations cannot be spatially correlated, it should be possible to remove them locally. (2) Local transformations devised for such purposes should preserve the local physical meaning of the gauge field, thus giving a reasonable expectation that only unphysical randomness is eliminated while physics is retained (together with the randomness that is necessary). The first candidate transformations of this kind are the chiral ordering transformations of Ref. [1]. Given a chirally symmetric Dirac operator $D$, the prototypical chiral ordering transformation replaces the link $U_{n, \mu}$ with an SU(3) element $\bar{U}_{n, \mu}$ minimizing the norm of the matrix [1]

$$
D_{n, n+\mu}(U)-D_{\mu}^{f} \times \bar{U}_{n, \mu} \quad \text { where } \quad D_{\mu}^{f} \equiv \frac{1}{3} \operatorname{tr}^{c} D_{n, n+\mu}(\mathbb{I})
$$

In other words, the transformed gauge connection represents an effective matrix phase by which fermionic variables get rotated under hopping from $n+\mu$ to $n$, relative to the free hopping. This preserves the local interpretation of the gauge field as a "phase rotator" for the charged particle interacting with it. Given the expected non-ultralocality of $D$ in gauge variables, the above transformations are non-trivial for generic backgrounds.

Using the locality of chiral ordering transformations, one can argue that applying them repeatedly at the ensemble level leads to the evolution in the set of valid gauge actions [1]. The theories reached this way are expected to generate more space-time order (lower Kolmogorov entropy) in their representative configurations than the starting theory. The characteristic feature of corresponding actions is that they become functions of the chirally symmetric Dirac kernel $D$ on which the transformation is based. This fact suggests a tempting possibility that, at least for the purposes of studying the QCD vacuum structure, it would be interesting to explicitly construct lattice regularizations where both gauge and fermionic parts of the theory enter in a unified manner, namely via lattice Dirac operator with exact chiral symmetry [3]. In fact, such coherence can be 
extended to all elements of the theory including the operators for relevant observables. In this talk we discuss some aspects of this construction, given fully in Ref. [3], with the particular focus on arriving at the formulation where gauge and fermionic parts of the full action are cast in the most mutually symmetric form.

\section{Simple Coherent Lattice QCD}

The simplest version of lattice QCD (LQCD) where all parts of the action are constructed from $D$ (coherent $L Q C D)$, is based on the following conjecture [3] ${ }^{1}$

Conjecture C3. Let $A_{\mu}(x)$ be arbitrary smooth su(3) gauge potentials on $\mathbb{R}^{4}$. If $U(a) \equiv\left\{U_{n, \mu}(a)\right\}$ is the transcription of this field to the hypercubic lattice with classical lattice spacing $a$, and $\mathbb{I} \equiv$ $\left\{U_{n, \mu} \rightarrow \mathbb{I}^{c}\right\}$ is the free field configuration then

$$
\operatorname{tr}\left(D_{0,0}(U(a))-D_{0,0}(\mathbb{I})\right)=-c^{S} a^{4} \operatorname{tr} F_{\mu \nu}(0) F_{\mu v}(0)+\mathscr{O}\left(a^{6}\right)
$$

for generic chirally symmetric $D$. Here $c^{S}$ is a non-zero constant independent of $A_{\mu}(x)$ at fixed $D$, and $F_{\mu v}(x) \equiv \partial_{\mu} A_{v}(x)-\partial_{v} A_{\mu}(x)+\left[A_{\mu}(x), A_{v}(x)\right]$ is the field-strength tensor.

The heuristic reason for validity of the above conjecture is that $\operatorname{tr} D_{n, n}$ is scalar, local, gauge invariant function of the gauge field. Up to dimension four, the only possibilities for such operators in the continuum are the constant and $\operatorname{tr} F_{\mu v}(x) F_{\mu v}(x)$. For the family of standard overlap Dirac operators based on the Wilson-Dirac operator with mass $-\rho$ [4], the validity of Conjecture C3 will be supported by both analytical and numerical methods in Ref. [5], where the constants $c^{S}(\rho)$ will also be evaluated. Note that the conjecture analogous to $\mathrm{C} 3$ in the pseudoscalar case is in fact a basis for constructing topological density from chirally symmetric Dirac operator [6] (see also Ref. [7]). For overlap Dirac operator this was examined explicitly in Refs. [8].

Accepting the validity of Conjecture $\mathrm{C} 3$ allows us to define the action for simplest version of coherent LQCD with $N_{f}$ flavors of quarks and the CP-violating $\theta$-term as [3]

$$
S_{\bar{\beta}, \bar{\theta},\left\{m_{f}\right\}}(U)=\operatorname{Tr}\left(\bar{\beta}-i \bar{\theta} \gamma_{5}\right)(D(U)-D(\mathbb{I}))+\sum_{f=1}^{N_{f}} \bar{\psi}^{f}\left(D(U)+m_{f}\right) \psi^{f}
$$

where $\left\{m_{f}\right\}$ is the set of real non-negative quark masses, and

$$
\bar{\beta}(\beta)=\frac{\beta}{12 c^{S}} \quad\left(\beta \equiv \frac{6}{g^{2}}\right) \quad \bar{\theta}(\theta)=\frac{\theta}{16 \pi^{2} c^{P}} \quad(\theta \in(-\pi, \pi])
$$

The constant $c^{P}$ appearing in pseudoscalar term is defined analogously to $c^{S}$. We note that the locality of $S$ defined above follows from locality of $D$, and that the free-field term in the gauge part of the action only contributes a field-independent constant which can be discarded if desired.

\footnotetext{
${ }^{1}$ Note that we will keep both the notation and the numbering of conjectures the same as in Refs. [1, 3]. Also, the local traces are denoted by "tr" while the global ones by "Tr" and, unless denoted explicitly by a superscript, the local traces are taken over the full linear space appropriate for the object in question.
} 
The dynamics of coherent QCD (2.2) is completely encoded in the chirally symmetric lattice Dirac operator. This becomes more explicit after fermionic variables are integrated out. In case of degenerate quark masses the distribution density of the gauge fields is given by

$$
P_{\bar{\beta}, \bar{\theta}, m}(U) \propto e^{\operatorname{Tr}\left[N_{f} \ln (D+m)+\left(-\bar{\beta}+i \bar{\theta} \gamma_{5}\right) D\right]}=\operatorname{det}\left[(D+m)^{N_{f}} e^{\left(-\bar{\beta}+i \bar{\theta} \gamma_{5}\right) D}\right]
$$

where $D \equiv D(U)$. Thus, for $\theta=0$ this formulation requires simulating the probability distribution

$$
P_{\bar{\beta}, m}(U) \propto \operatorname{det}\left[(D+m)^{N_{f}} e^{-\bar{\beta} D}\right]=\operatorname{det}\left[\left(-\frac{d}{d \bar{\beta}}\right)^{N_{f}} e^{-\bar{\beta}(D+m)}\right]
$$

where the last equality emphasizes the close explicit relation between fermionic and gauge contributions to the full probability distribution. Some initial ideas on simulating this theory based on overlap Dirac kernel are presented in Ref. [9].

\section{Symmetric Logarithmic LQCD}

It is easy to see that the simple coherent LQCD discussed above is by no means the only possibility for constructing lattice regularizations where the gauge and fermionic parts of the action are tied together in an explicit manner. To obtain more general class of such formulations, we will treat the Dirac operator $D$ defining fermionic action as a primary object, and discuss possibilities for forming the gauge parts of the action from various functions $f(D)$. In particular, if $f(D)$ is a local operator and if $\operatorname{tr} f(D)_{n, n}$ is a scalar lattice field, then we generically expect the validity of a statement analogous to Conjecture C3 with equation (2.1) replaced by

$$
\operatorname{tr}\left[f(D(U(a)))_{0,0}-f(D(\mathbb{I}))_{0,0}\right]=-c^{S} a^{4} \operatorname{tr} F_{\mu \nu}(0) F_{\mu \nu}(0)+\mathscr{O}\left(a^{6}\right)
$$

where $c^{S}$ is an associated constant. Proceeding in the same way also for the pseudoscalar case (i.e. considering operator $\gamma_{5} f(D)$ ) we obtain the definition of coherent LQCD in this case by replacing $D$ in the gauge part of Eq. (2.2) with $f(D)$, while Eq. (2.3) remains unchanged. Some attractive choices for functions $f(D)$ include $D^{k}, \ln (D+\eta)$, and $(D+\eta)^{-1}[3]$.

We will now focus on constructing coherent LQCD in which the gauge and fermionic contributions to the action enter in the most mutually symmetric manner. Since the fermionic part is fixed, we can bring the two forms closer together if we mimic the effective fermionic action (after integrating out fermions) in the construction of the gauge part, i.e. if we use $f(D)=\ln (D+\eta)$. This leads to

$$
S_{\bar{\beta}, \bar{\theta},\left\{m_{f}\right\}}(U)=\operatorname{Tr}\left(\bar{\beta}-i \bar{\theta} \gamma_{5}\right) \ln \left(D(U)+m_{0}\right)+\sum_{f=1}^{N_{f}} \bar{\psi}^{f}\left(D(U)+m_{f}\right) \psi^{f}
$$

where we denoted $\eta \equiv m_{0}>0$ to reflect its mass-like form. Note however that, unlike the lattice quark masses $m_{f}$, the parameter $m_{0}$ is kept fixed as the continuum limit is approached. In effect, different values of $m_{0}$ control the lattice locality range of the gauge action. We refer to the above formulation as logarithmic LQCD [3]. For $\theta=0$ and degenerate quark masses, the probability distribution of gauge fields to simulate in this case is

$$
P_{\bar{\beta}, m}(U) \propto e^{\operatorname{Tr}\left[N_{f} \ln (D+m)-\bar{\beta} \ln \left(D+m_{0}\right)\right]}=\operatorname{det}\left[(D+m)^{N_{f}}\left(D+m_{0}\right)^{-\bar{\beta}}\right]
$$


Interestingly, it is possible to bring the gauge action to the form that is yet more (and completely) analogous to the effective action of a single lattice fermionic flavor. To see that, consider the effective action of logarithmic LQCD with one flavor and $\theta=0$, namely

$$
-S_{m, \bar{\beta}}^{e f f}(U)=\operatorname{Tr}\left[\ln (D+m)-\bar{\beta} \ln \left(D+m_{0}\right)\right] \quad \bar{\beta} \equiv \frac{1}{2 g^{2} c^{S}\left(m_{0}\right)}
$$

We can thus eliminate varying $\bar{\beta}$ in favor of varying $m_{0}$ (and bring the two terms into almost identical form), if for arbitrary $g>0$ from some finite vicinity of $g=0$, we can find unique $m_{0} \equiv$ $m_{0}(g)$ such that $\left|c^{S}\left(m_{0}\right)\right| \equiv 1 / 2 g^{2}$. This is indeed expected to be possible since $\ln \left(D+m_{0}\right)_{n, n}$ will diverge as $m_{0} \rightarrow 0$ for smooth configurations. A precise statement can be formulated as follows [3].

Conjecture C6. Let $D$ be a chirally symmetric operator such that $f(D) \equiv \ln (D+\eta)$ is well-defined for arbitrary $\eta>0$. If $c^{S}(\eta)$ is the associated classical coupling of $\operatorname{tr} f(D(U))_{n, n}$ to $\operatorname{tr} F_{\mu \nu} F_{\mu \nu}$, then there exists $\eta_{0}>0$ such that $c^{S}(\eta)$ is monotonic for $0<\eta \leq \eta_{0}$, and $\lim _{\eta \rightarrow 0}\left|c^{S}(\eta)\right|=\infty$. Moreover, there exists a non-zero (possibly infinite) limit

$$
\lim _{\eta \rightarrow 0} \frac{c^{S}(\eta)}{\ln (\eta)} \equiv \lim _{\eta \rightarrow 0} \kappa^{S}(\eta) \equiv \kappa^{S}(0) \neq 0
$$

Conjecture C6 implies the existence of a one-to-one correspondence between $g \in\left(0, g_{0}\right]$ and $m_{0} \in$ $\left(0, \eta_{0}\right]$, such that $\left|c^{S}\left(m_{0}\right)\right| \equiv 1 / 2 g^{2}$. We thus have instead of (3.4)

$$
-S_{m, m_{0}}^{e f f}(U)=\operatorname{Tr}\left[\ln (D+m)-\operatorname{sgn}\left(c^{S}\left(m_{0}\right)\right) \ln \left(D+m_{0}\right)\right] \quad\left|c^{S}\left(m_{0}\right)\right| \equiv \frac{1}{2 g^{2}}
$$

The full-fledged form of the lattice theory constructed according to the above arguments is [3]

$$
\begin{aligned}
S_{m_{0}^{S}, m_{0}^{P},\left\{m_{f}\right\}} & =\operatorname{sgn}\left(c^{S}\left(m_{0}^{S}\right)\right) \operatorname{Tr} \ln \left(D+m_{0}^{S}\right)-i \operatorname{sgn}\left(c^{P}\left(\left|m_{0}^{P}\right|\right)\right) \operatorname{sgn}\left(m_{0}^{P}\right) \operatorname{Tr} \gamma_{5} \ln \left(D+\left|m_{0}^{P}\right|\right) \\
& +\sum_{f=1}^{N_{f}} \bar{\psi}^{f}\left(D+m_{f}\right) \psi^{f}
\end{aligned}
$$

where the "gauge" parameters $m_{0}^{S}, m_{0}^{P}$ are related to $g$ and $\theta$ via

$$
\left|c^{S}\left(m_{0}^{S}\right)\right| \equiv \frac{1}{2 g^{2}} \quad \theta=\operatorname{sgn}\left(m_{0}^{P}\right) 16 \pi^{2}\left|c^{P}\left(\left|m_{0}^{P}\right|\right)\right| \quad \theta \in(-\pi, \pi]
$$

and the various mass-like lattice parameters of the theory vary within the ranges

$$
m_{f} \in(0, \infty) \quad m_{0}^{S} \in\left(0, m_{0}^{S, c}\right] \quad m_{0}^{P} \in\left[m_{0}^{P, c}, \infty\right) \cup\left(-\infty,-m_{0}^{P, c}\right)
$$

In the above equations, $m_{0}^{S, c}$ is the maximal $\mu_{0}$ satisfying the statement of Conjecture C6. Note that in this formulation we have allowed the pseudoscalar mass $m_{0}^{P}$ to be negative so that the lattice action density preserves exactly the transformation property of the pseudoscalar part under $\theta \rightarrow$ $-\theta$. Such definition assumes that $\left|c^{P}(\eta)\right|$ vanishes at infinity and is monotonically decreasing in the range $\eta \in\left[m_{0}^{P, c}, \infty\right)$, where $m_{0}^{P, c}$ is defined via $\theta\left(m_{0}^{P, c}\right) \equiv \pi=16 \pi^{2}\left|c^{P}\left(m_{0}^{P, c}\right)\right|$. For the family of overlap Dirac operators $D^{(\rho)}$ this is satisfied with $m_{0}^{P, c}=\frac{2 \rho}{e^{\pi}-1}$. Lattice regularization constructed 
above is referred to as symmetric logarithmic LQCD [3]. The continuum limit with $N_{f} \leq 16$ is taken via $m_{0}^{S} \equiv m_{0}^{S}(a) \rightarrow 0$ while decreasing $m_{f}=m_{f}(a) \propto a \bar{m}_{f}^{r}$ towards zero so that some set of renormalized masses $\bar{m}_{f}^{r}$ (in physical units) is held fixed, and with the pseudoscalar mass $m_{0}^{P}$ (specifying $\theta$ ) kept unchanged in the process.

From Eq. (3.6) one can see that the gauge action in symmetric logarithmic LQCD can be viewed as an effective action of a lattice fermion or pseudofermion, depending on the sign of $c^{S}(\eta)$ in the vicinity of $\eta=0$. Preliminary calculations indicate [5] that, for overlap Dirac operator, $c^{S}(\eta)<0$ sufficiently close to $\eta=0$, and this is expected to be true in general. In this case, the effective action of symmetric logarithmic LQCD at $\theta=0$ can be written as

$$
-S_{\left\{m_{f}\right\}}^{e f f}=\operatorname{Tr} \sum_{f=0}^{N_{f}} \ln \left(D(U)+m_{f}\right)=\operatorname{Tr} \ln \prod_{f=0}^{N_{f}}\left(D(U)+m_{f}\right)
$$

with the gauge contribution to the action entering at the regularized level in a completely formsymmetric manner relative to the contribution of a single fermionic flavor. Introducing the Grassmann variables for this " 0 -th flavor" of mass $m_{0} \equiv m_{0}^{S}$, the total action can be written in the form

$$
S_{\left\{m_{f}\right\}}=\sum_{f=0}^{N_{f}} \bar{\psi}^{f}\left(D(U)+m_{f}\right) \psi^{f}
$$

Thus, symmetric logarithmic LQCD casts the regularized dynamics of full QCD into that of $N_{f}+1$ lattice fermionic flavors interacting with $\mathrm{SU}(3)$ gauge field. While this might sound suspicious at first, in fact it is not. It turns out that what distinguishes "gauge" from "fermionic" in this case is the locality of corresponding effective operators in the continuum limit. Indeed, since for the " $0-$ th" flavor the corresponding effective operator is the gauge action itself, we have to insist that its effective range in physical units shrinks to zero in the continuum limit ("weak locality" [3]). For theory with $N_{f}$ asymptotically free flavors this translates into the requirement [3]

$$
0=\lim _{a \rightarrow 0} \frac{a}{m_{0}(a)} \propto \lim _{m_{0} \rightarrow 0} m_{0}^{\frac{\left|\kappa^{S}(0)\right|}{\beta_{0}}-1} \quad \text { or } \quad \frac{\left|\kappa^{S}(0)\right|}{\beta_{0}}>1
$$

where $\beta_{0}=\left(11-\frac{2}{3} N_{f}\right) / 16 \pi^{2}$. The above condition can also be viewed as a requirement on the number of asymptotically free flavors for which QCD can be defined via symmetric logarithmic LQCD, namely that $N_{f}>\frac{33}{2}-24 \pi^{2}\left|\kappa^{S}(0)\right|$. Thus, if this condition is satisfied, then the range of the effective action for the " 0 -th" (gauge) flavor is zero in the continuum limit, while the ranges of effective actions corresponding to usual quark flavors are non-zero and inversely proportional to the corresponding renormalized quark masses $\bar{m}_{f}^{r}$. This is equivalent to saying that the " 0 -th" flavor in (3.11) is infinitely heavy in the continuum limit. Indeed, from Eq. (3.12) we have that its mass in physical units is proportional to

$$
\lim _{a \rightarrow 0} \frac{m_{0}(a)}{a} \propto \lim _{m_{0} \rightarrow 0} m_{0}^{1-\frac{\left|\kappa^{S}(0)\right|}{\beta_{0}}}=\infty
$$

Consequently, all correlators involving the variables $\psi_{0}, \bar{\psi}_{0}$ in theory (3.11) are expected to vanish in the continuum limit, and the " $0-$ th" flavor decouples from the light quark flavors. 


\section{Discussion}

In this talk we have argued that, for the purposes of studying QCD vacuum structure, it might be fruitful to explore lattice regularizations of QCD where gauge and fermionic aspects of the theory are both based on a lattice Dirac operator $D$ with exact chiral symmetry (coherent LQCD) [3]. The motivations associated with vacuum structure are mostly related to the relevance of chiral ordering transformations of Ref. [1]. However, it is quite clear that this novel approach to constructing lattice regularizations might be interesting in its own right. Indeed, the explicit interrelations between gauge and fermionic aspects of the theory, present in such regularizations, potentially offer valuable insights into the "inner workings" of QCD dynamics. After all, the properties of valid regularizations become properties of QCD in the continuum limit. Following this route, the goal of our discussion was to arrive at the formulation where gauge and fermionic parts of the full action become mutually form-symmetric to a maximal possible degree. Starting from simplest coherent LQCD and its generalizations, we arrived at symmetric logarithmic LQCD, where quarks and gluons contribute to an overall dynamics in a completely form-symmetric manner [3]. This can be most clearly seen in Eq. (3.11), where the gauge part of the dynamics is represented via an additional fermionic flavor that becomes infinitely heavy in the continuum limit.

The existence of lattice regularizations where gauge dynamics can be viewed as inherited from infinitely heavy fermions (in logarithmic LQCD there are in fact infinitely many of them in the continuum limit [3]) raises a question if QCD has a "natural definition" from the point of view of the theory beyond the Standard Model. Indeed, if the heavy decoupled particles in question can be given physical meaning in such context, then this possibility might acquire some content.

Finally, we wish to mention that the "coherence" in definition of LQCD can be extended to include all operators by application of chiral ordering transformations [1,3]. Some interesting operators can also be constructed from $D$ explicitly $[3,9,5]$.

Acknowledgment: Numerous discussions with Andrei Alexandru and Keh-Fei Liu on the topics related to the subject of this talk are gratefully acknowledged.

\section{References}

[1] I. Horváth, hep-lat/0605008.

[2] I. Horváth et al., Phys. Rev. D68, 114505 (2003).

[3] I. Horváth, hep-lat/0607031.

[4] H. Neuberger, Phys. Lett. B417 (1998) 141; Phys. Lett. B427 (1998) 353.

[5] A. Alexandru, I. Horváth, K.F. Liu, in preparation.

[6] P. Hasenfratz, V. Laliena, F. Niedermayer, Phys. Lett. B427 (1998) 125.

[7] R. Narayanan and H. Neuberger, Nucl. Phys. B443, 305 (1995).

[8] Y. Kikukawa and A. Yamada, Phys. Lett. B448 (1999) 265; K. Fujikawa, Nucl. Phys. B546 (1999) 480; D. Adams, Annals Phys. 296 (2002) 131; H. Suzuki, Prog. Theor. Phys. 102 (1999) 141; D. Adams, J. Math. Phys. 42 (2001) 5522; W. Kerler, Int. J. Mod. Phys. A16 (2001) 3117.

[9] K.F. Liu, these proceedings, [hep-lat/0609033]; K.F. Liu, A. Alexandru, I. Horváth, in preparation. 